\title{
Chronic Brucella infection of the supra-patellar bursa with sinus formation
}

\author{
M McDermott, B O’Connell, T E Mulvihill, E C Sweeney
}

\begin{abstract}
A case of supra-patellar bursitis with the formation of discharging sinus is described. $B$ abortus was isolated from resected bursal tissue. While osteoarticular complications of brucellosis are common and a number of different clinical syndromes are now recognised, disease of the bursae is rare and as far as is known sinus formation has not been described before in this setting.
\end{abstract}

(F Clin Pathol 1994;47:764-766)

\section{Case report}

A 39 year old man presented with a small infected skin lesion above the left knee, which was treated successfully with flucloxacillin. He presented four months later with a recurrence of the lesion and associated inguinal adenopathy and fever. A full blood count was normal and the monospot test was negative, but Brucella sp antibody serology titres were increased with a Coombs test result of 1/5120. A tentative diagnosis of Brucella infection was made, although the exact nature of the skin lesion remained unclear. There was no history of occupational exposure to farm animals, but the patient had occasionally consumed unpasteurised milk from neighbouring farms.

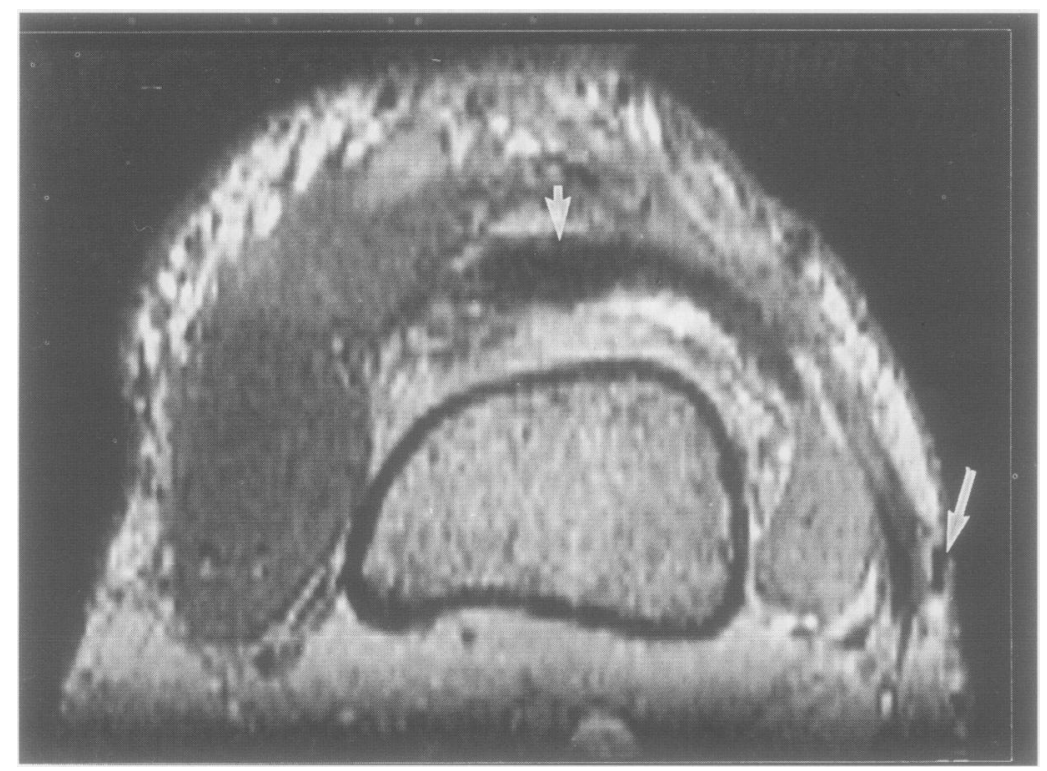

Figure 1 Axial magnetic resonance image scan of knee demonstrating fluid filled space (short arrow) anterior to lower end of the femur with lateral subcutaneous extension to the site of the discharging sinus (long arrow).
Three years later, he developed a discharging sinus which required surgical excision of a blind-ending subcutaneous tract above the left knee. There was no obvious infection in the knee joint. Histological examination of the resected material confirmed the presence of a sinus tract, lined by granulation tissue with adjacent chronic inflammation and necrosis. The features were felt to represent an inflamed bursa and the patient was discharged without additional treatment.

Seven years after initial presentation, a further purulent discharging sinus developed at the same site. Clinically, erythema, oedema, and tenderness were present in the area but, as on previous admission, there was no limitation of movement. Culture of the purulent exudate grew no organisms. Repeat Brucella serology using $B$ abortus antigen (Murex Diagnostics, Hartford, England) produced a standard agglutination test of $1 / 1280$ and a Coombs test result of $1 / 5120$. Magnetic resonance imaging demonstrated a multioculated soft tissue swelling above and anterior to the knee (fig 1). Surgical exploration was undertaken with drainage of a multiloculated abscess and resection of large quantities of granulation tissue from the soft tissues around the knee. Histologically, the resection specimen consisted of chronically inflamed hyperplastic synovium with surface fibrinous exudate. No lymphoid aggregates were identified, but there were numerous, poorly formed necrotising granulomata in the surrounding connective tissue (fig 2). Ziehl-Neelsen and Gram stains were negative. Culture of resected material, after three weeks of incubation, grew Brucella abortus biotype 1. The patient was started on oral tetracycline 500 mg four times a day and has made a good recovery.

\section{Discussion}

Brucella infection is associated with arthritis in $10-25 \%$ of patients ${ }^{2}$ and may precede, accompany, or follow systemic infection. ${ }^{3}$ Four clinical patterns of joint disease are described, listed in order of frequency: (i) sacroiliitis; (ii) peripheral arthritis, usually monoarticular, affecting knee, hip, ankle and shoulder; (iii) mixed forms; (iv) and spondylitis, the most frequently destructive form. ${ }^{4}$ Arthritis may be seen in association with acute, undulant, or chronic systemic disease. ${ }^{5}$ Spondylitis is strongly associated with chronic disease and tends to occur in a more elderly population, while sacroiliitis and peripheral arthritis are more frequently acute or subacute 


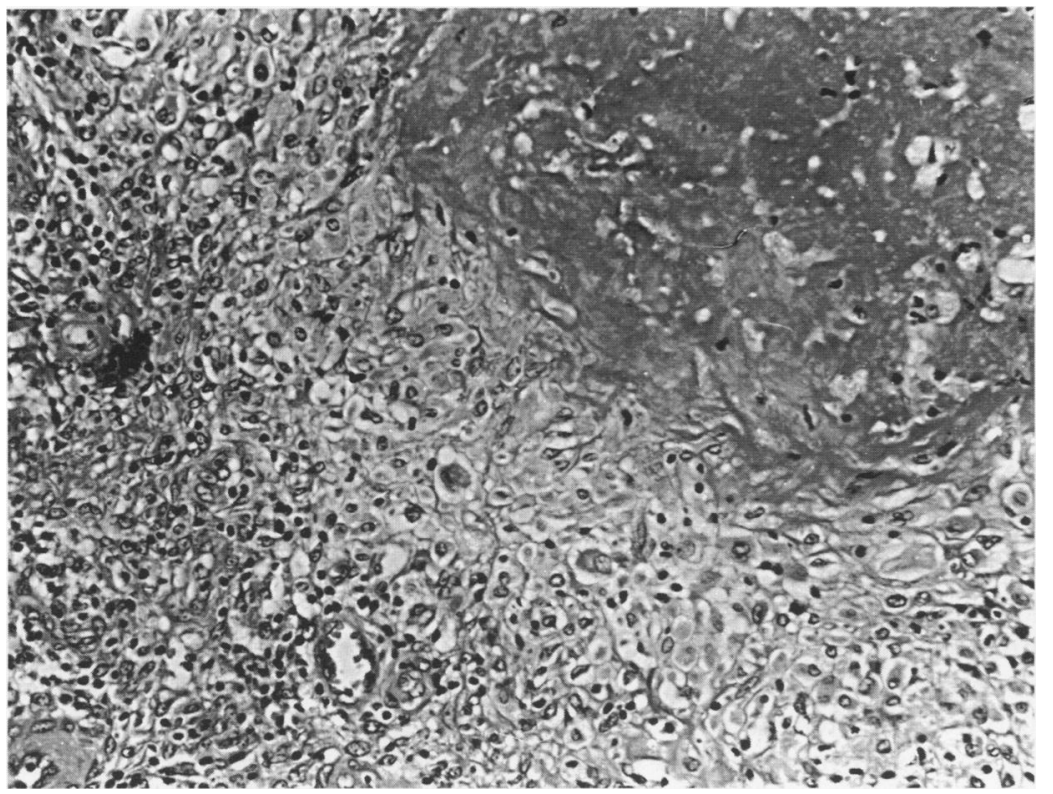

Figure 2 Section of resected bursa showing severe chronic inflammation and a large necrotising granuloma.
The isolation of Brucella spp, in particular $B$ abortus, from synovial fluid is difficult (authors quoting success rates of $35-92 \%),{ }^{34}$ and depends to a large extent on a clinical suspicion and a high level of vigilance at bench level. This difficulty may be due to the relative paucity of organisms present in clinical specimens as it has been shown that apart from $B$ abortus biotype 2 and $B$ suis biotype 3 brucellae are not fastidious organisms. ${ }^{10}$ Various media are suitable for Brucella isolation including chocolate agar, which was successful in this case.

Because of the poor yield from culture of $B$ abortus, which is almost invariably the pathogen in the United Kingdom, many rely on a combination of clinical presentation and serological tests to arrive at a diagnosis. The standard agglutination test is the most widely used serological proceduri, with a titre of $>$ $1 / 160$ considered by many to be diagnostic in acute infection. However, these tests need to be interpreted with caution as no single titre may be taken to indicate active disease, especially in a rural population where a significant degree of exposure and subclinical infection occurs. In the Republic of Ireland, where brucellosis remains endemic in cattle, it has been shown that over $40 \%$ of blood donors in a rural community have standard agglutination titres of $>1 / 80$, whereas only $14 \%$ of blood donors from an urban community have similar titres (personal communication, $\mathrm{Mr}$ Liam English, Department of Microbiology, St James's Hospital, and unpublished data).

Histological changes associated with Brucella infection of synovial lined spaces are variable but are principally those of a chronic inflammation, with hyperplasia of lining cells. Lymphocytes, plasma cells, and histiocytes are the predominant cell types, with occasional giant cells and granuloma formation. ${ }^{1}$ Granulomata may undergo central necrosis ${ }^{1}$ but are sparse and therefore infrequently found. ${ }^{46}$ Some authors feel that the non-specific histological picture means that synovial biopsy is not a diagnostically useful procedure in this setting. ${ }^{2}$ However, the finding of granulomata significantly narrows the range of diagnostic possibilities and, if not pathognomonic of Brucella infection, may at least add further weight to an established clinical suspicion. As such, biopsy will continue to have a role, particularly where the diagnosis has proved difficult. Biopsy also provides material for immunocytochemical identification of Brucella organisms. Immunoperoxidase antibodies are commercially available (Wellcome) and are used extensively in the veterinary industry. However, the small numbers of organisms typically associated with human disease mean that the diagnostic yield will be low and in this case no organisms were identified using this method.

The high percentage of cases in which Brucella has not been cultured from synovial fluid has been used as an argument for the existence of a "reactive" Brucella arthritis as well as an "infective" form. ${ }^{5}$ This suggestion is supported by the non-specific histological automated or semi-automated methodology increase the yield from blood cultu Lysis-centrifugation has been reported being more successful than the Castaneda method ${ }^{11}$ and similarly continuous monitoring is promising. ${ }^{12}$ 
picture, the non-destructive nature of many of these infections, and the resolution of cases without anti-microbial treatment. ${ }^{5}$ Many authors have also documented the existence of immunological abnormalities in Brucella arthritis, including IgG agglutinating antiBrucella antibodies in synovial fluid of such patients, and point to the similarity of the condition with other "reactive" arthritides such as those following Shigella and Yersinia infection. ${ }^{4}$ However, isolation of organisms from the joint in such cases, improved by using appropriate media and conditions, immediately moves cases of otherwise typical "reactive" arthritis into the "infective" category. Further improvements in organism detection are likely to follow the application of molecular biological techniques to the field, such as PCR amplification of Brucella DNA.

The current case illustrates the lengthy delay in reaching a firm diagnosis in musculoskeletal brucellosis. The rarity of the condition, the "non-infectious" synovial fluid picture, and the difficulty in culturing the organism all militate against prompt diagnosis and treatment. ${ }^{4}$ These factors were compounded in this case by the absence of any of the more traditional risk factors for the illness, such as farming or abattoir work (the patient had had a number of occupations, but was a courier at the time of initial presentation). The predominant bursal location of the inflammatory process without evidence of direct articular disease which allowed normal range of movement throughout the seven year history of the lesion was also perplexing. Bursal disease is uncommon, with the three cases described by Johnson and Weed representing the largest series to date. Bursitis and tendinitis were not separated as clinical syndromes in the series reported by Mousa et al, but the combination represented only $1 \cdot 2 \%$ of the 169 cases of osteoarticular brucellosis they described, and bursitis is not recorded in other large series. ${ }^{23}$ The formation of a sinus tract in this setting has also not been described before.

We acknowledge the technical assistance of Margaret Lynch Department of Microbiology, St James' Hospital.

1 Walker AN, Fechner RE. Granulomatous inflammation of bones and joints. Pathology of granulomas, New York Raven Press 1983: 421-48.

2 Khateeb MI, Araj GF, Majeed SA, Lulu AR. Brucella arthritis: A study of 96 cases in Kuwait. Ann Rheum Dis 1990;49:994-8

3 Lulu AR, Araj GF, Khateeb MI, Mustafa MY, Yusuf AR, Fenech FF. Human brucellosis in Kuwait: A prospective study of 400 cases. $Q \mathcal{F}$ Med $1988 ; 249: 39-54$

4 Gotuzzo E, Alarcon GS, Bocanegra TS, Carrillo C, Guerra JC, Rolando I, et al. Articular involvement in human brucellosis: A retrospective analysis of 304 cases. Semin Arthritis Rheum 1982;12:245-55.

5 Alarcon GS, Bocanegra TS, Gotuzzo E, Espinoza LR. The arthritis of brucellosis: A perspective one hundred years after Bruce's discovery. $\mathcal{F}$ Rheumatol 1987;14:1083-5.

6 Johnson WE, Weed LA. Brucellar bursitis. $\mathcal{F}$ Bone fnt Surg 1954;364:133-41.

7 Young EJ. Human brucellosis. Rev Infect Dis 1983 $5: 821-42$.

8 Mousa ARM, Mutaseb SA, Almudallal DS, Khodeir SM Marafie AA. Osteoarticular complications of brucellosis: A study of 169 cases. Rev Infect Dis 1987;9:531-43.

9 Spink WW. The nature of brucellosis. Minneapolis: university of Minnesota Press, 1956:145-90.

10 Robertson L, Farrell ID, Hinchcliffe PM, Quaife RA. Benchbook on Brucella. PHLS Monograph Series, London: HMSO: 1980.

11 Etamadi H, Raissadat A, Pickett MJ, et al. Isolation of Brucella spp from clinical specimens. $\mathcal{f}$ Clin Microbio 1984;20:586.

12 Solomon HM, Jackson D. Rapid diagnosis of Brucella melitensis in blood: some operational characteristics of the BACT/ALERT. $f$ Clin Microbiol 1992;30:222-4.

\section{Cushing's syndrome associated with recurrent endometrioid adenocarcinoma of the ovary}

\author{
Cancer Medicine \\ Research Unit \\ University of Bradford \\ and Department of \\ Oncology, Airedale \\ General Hospital, \\ Keighley \\ S M Crawford \\ Department of \\ Histopathology, \\ Airdale General \\ Hospital \\ R D Pyrah \\ Department of \\ Pathology, University \\ of Wales College of \\ Medicine, Cardiff \\ S M Ismail \\ Correspondence to \\ Dr S M Crawford, Cancer \\ Medicine Research Unit, \\ University of Bradford, \\ Bradford, West Yorkshire \\ BD7 1DP \\ Accepted for publication \\ 14 December 1993
}

\author{
S M Crawford, R D Pyrah, S M Ismail
}

\begin{abstract}
Ectopic production of adrenocorticotrophic hormone (ACTH) by malignant neoplasms is a well recognised cause of Cushing's syndrome but is extremely rare in ovarian carcinoma. A patient who underwent surgery for ovarian carcinoma followed by a course of chemotherapy is reported. The tumour was a bilateral moderately differentiated endometrioid adenocarcinoma and contained numerous chromogranin immunoreactive endocrine cells as well as small foci of ACTH immunoreactivity. She subse-
\end{abstract}

quently presented with Cushing's syndrome in association with extensive pelvic recurrence of the tumour.

$(\Im$ Clin Pathol 1994;47:766-768)

Cushing's syndrome due to ectopic adrenocorticotrophic hormone (ACTH) production has been described in a wide range of ovarian tumours including sex cord stromal tumours, ${ }^{1}$ carcinoid tumours, ${ }^{1-3}$ and teratomas. ${ }^{4}$ However, ovarian tumours of common epithelial type are an extremely uncommon cause of 